\title{
Studies on the Structure-Activity Relationship and Mode of Actions of Neonicotinoid Insecticides*
}

\author{
Hisashi NISHIWAKI** \\ Faculty of Agriculture, Kinki University, 3327-204 Nakamachi, \\ Nara 631-8505, Japan
}

(Accepted May 21, 2004)

\begin{abstract}
The binding affinity of neonicotinoids for housefly head membranes was evaluated to elucidate the physicochemical features of the compounds involved in the ligand-nicotinic acetylcholine receptor (nAChR) interactions. Both steric and electrostatic factors of the substituents on the aromatic and imidazolidine rings were found to influence the binding affinity. $\quad\left[{ }^{14} \mathrm{C}\right]$ Imidacloprid was employed to clarify the metabolism of imidacloprid in the flies. It was found that the compound was degraded, and the metabolites were then excreted from the flies in a short period after administration. Agonist actions of neonicotinoids on nAChRs were evaluated to examine their relationships with the binding and insecticidal activities. High correlations were observed among these activities, suggesting that the channel opening of nAChRs resulting from the neonicotinoid binding is likely to cause the insecticidal actions.
\end{abstract}

Keywords: neonicotinoids, structure-activity relationship, metabolism, synergist, electrophysiology, housefly.

\section{INTRODUCTION}

Imidacloprid was developed by Bayer AG Co. Ltd. in the later 1980s. Bai et al. provided the first and direct evidence for the nicotinic agonist actions of imidacloprid. They showed that imidacloprid was able to depolarize an identified motor neuron Df of American cockroaches, which was blocked by $\alpha$ bungarotoxin. In addition, they showed that $\left[{ }^{125} \mathrm{I}\right] \alpha$-bungarotoxin binding to the cockroach membrane preparations was displaced by imidacloprid, suggesting that the insecticide was an agonist of insect nAChRs. Unlike nicotine, imidaclprid and related compounds selectively act on insects over vertebrates. Thus it was proposed that the insecticides should be referred to as neonicotinoids.

The agonist activities of several neonicotinoids on insect as well as vertebrate nAChRs have been evaluated using various electrophysiological methods. Also, the binding affinities of neonicotinoids for insect and vertebrate membrane preparations have been evaluated. As a result, it is clear that the selectivity of neonicotinoid insecticides for insects over vertebrates is attributable to their higher affinity for insect than vertebrate nAChRs. However, little is known about the structural factors of neonicotinoids determining the receptor-

\footnotetext{
* See Part II for the full Japanese article.

** To whom correspondence should be addressed.

E-mail: nc_nhisa@nara.kindai.ac.jp
}

binding affinity, and the relationships between in vitro (receptor binding and electrophysiological) and in vivo (insecticidal) activities.

In this study, the structure-activity relationships of neonicotinoids were investigated to clarify the physicochemical factors influencing the insect nAChR-neonicotinoid interactions. Furthermore, to elucidate the mode of actions of neonicotinoids, the metabolism of $\left[{ }^{14} \mathrm{C}\right]$ imidacloprid in houseflies and the correlations between the agonist activity on the recombinant nAChRs expressed in Xenopus laevis oocytes and the binding as well as insecticidal activities were also investigated.

\section{STRUCTURE-BINDING AFFINITY RELATION- SHIP ANALYSES OF NEONICOTINOID INSECTICIDES}

To elucidate the electrostatic and steric features of neonicotinoids determining the binding affinity for housefly head membranes, Okazawa et al. deployed Comparative Molecular Field Analysis (CoMFA), a three-dimensional quantitative structure-activity relationship method. CoMFA revealed that the electrostatic regions surrounding the nitroimino group of imidacloprid play an important role in determining the binding affinity. For the pyridine ring as well as $\mathrm{C} 4-\mathrm{C} 5$ and N3 positions of imidazolidine ring, however, much less information, compared with that for the nitroimino moiety, was obtained about physicochemical factors influencing the receptor bind- 
ing affinity. Thus, a series of substituted benzyl derivatives, $\mathrm{N} 3$-substituted imidacloprid analogs, and imidacloprid-related diaza compounds were synthesized and their binding affinity for housefly head membrane was measured using not only $\left[{ }^{125} \mathrm{I}\right] \alpha$-bungarotoxin but also $\left[{ }^{3} \mathrm{H}\right]$ imidacloprid as the radioligands. As the results, the steric as well as electrostatic factors were found to affect the interaction of neonicotinoids with the housefly nAChRs.

\section{METABOLISM IN HOUSEFLIES}

It was found that the pre-application of the synergists propargyl propyl phenylphosphonate (NIA16388, NIA) and piperonyl butoxide (PBO) enhanced the insecticidal activity of imidacloprid against houseflies and cockroaches. To elucidate the mechanism, the metabolism of imidacloprid in the housefly, the clearance rate of the metabolites from insect body and the effects of NIA and PBO on metabolism and clearance were investigated using ${ }^{14} \mathrm{C}$-labeled imidacloprid.

After injection of the labeled imidacloprid, most of the radioactivity was excreted within $24 \mathrm{hr}$. The major part of the radioactivity in the excreta as well as that in the insect body was accounted for by the olefin derivative of imidacloprid. The pre-application of synergists such as NIA and PBO suppressed the degradation of imidacloprid and delayed the clearance of insecticidal components from houseflies. These results suggested that the oxidative metabolism plays a key role in the insecticidal activity of imidacloprid.

\section{CORRELATION BETWEEN IN VITRO (BIND- ING AFFINITY AND ELECTROPHYSIOLOGICAL) AND IN VIVO (INSECTICIDAL) ACTIVITIES}

Taking into consideration the results obtained by studying the metabolism of imidacloprid in the houseflies, the insecticidal (in vivo) activity was evaluated against the housefly Musca domestica under synergistic conditions with NIA and PBO, and the correlation between the binding and insecticidal activities was analyzed. As the result, a high correlation was seen between these activities, suggesting that the receptor binding causes the insecticidal actions. However, it was not clear whether the receptor binding of neonicotinoids such as the substituted benzyl derivatives induce the agonist action on nAChRs like imidacloprid, and whether the insecticidal activity is the result of such agonist actions. Thus, the agonist activity of neonicotinoids on the fruit fly $\mathrm{SAD} /$ chicken $\beta_{2}$ hybrid nAChRs expressed in Xenopus laevis oocyte was measured by two-electrode voltage-clamp electrophysiology.

Most neonicotinoids tested were capable of activating the $\mathrm{SAD} / \beta_{2}$ recombinant $\mathrm{nAChRs}$, inducing inward currents. Variations in this agonist activity were closely related to those in the binding and insecticidal activities. These findings suggested that the channel opening of the hybrid nAChR is likely to be induced by binding of the neonicotinoid ligands to the receptor, resulting in death of the insects. 